\title{
Tapinarof Cream 1\% Once Daily for Plaque Psoriasis: Efficacy by Baseline Disease Characteristics and Demographics in Two Pivotal Phase 3 Trials
}

Leon Kircik, ${ }^{1,2}$ Linda Stein Gold, ${ }^{3}$ James Del Rosso, ${ }^{4}$ Seemal R. Desai, ${ }^{5,6}$ Brad P. Glick, ${ }^{7}$ Howard Sofen, ${ }^{8}$ Anna M. Tallman, ${ }^{9}$ David S. Rubenstein, ${ }^{9}$ Philip M. Brown ${ }^{9}$

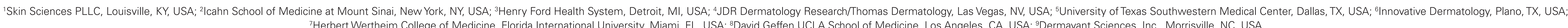

\section{SYNOPSIS}

Tapinarof is a first-in-class, non-steroidal, topical therapeutic aryl hydrocarbon receptor modulating agent (TAMA) in development for the treatment of psoriasis and atopic dermatitis

In two identical, randomized, double-blind Phase 3 trials, PSOARING and PSOARING 2, tapinarof cream 1\% once daily (OD) demonstrated highly statistically significant efficacy versus vehicle at 12 weeks and was well tolerated in adults with mild to severe plaque psoriasis'

\section{OBJECTIVE}

To present results for the pivotal Phase 3 primary efficacy endpoint (proportion of patients who achieved a Physician Global Assessment [PGA] response at Week 12) by baseline disease characteristics and demographics using pooled data from PSOARING 1 and PSOARING 2

\section{METHODS}

\section{Study Design}

Patients with mild to severe plaque psoriasis were randomly assigned

2:1 to receive tapinarof cream $1 \%$ OD or vehicle OD for 12 weeks in

two identical, Phase 3, multicenter (US and Canada), double-blind,

vehicle-controlled trials (Figure 1)

Following the double-blind period, patients could enroll in a separate

open-label, long-term extension study for an additional 40 weeks

of treatment, or complete a follow-up visit 4 weeks after the end of

treatment (Week 16)

Figure 1. Study Design

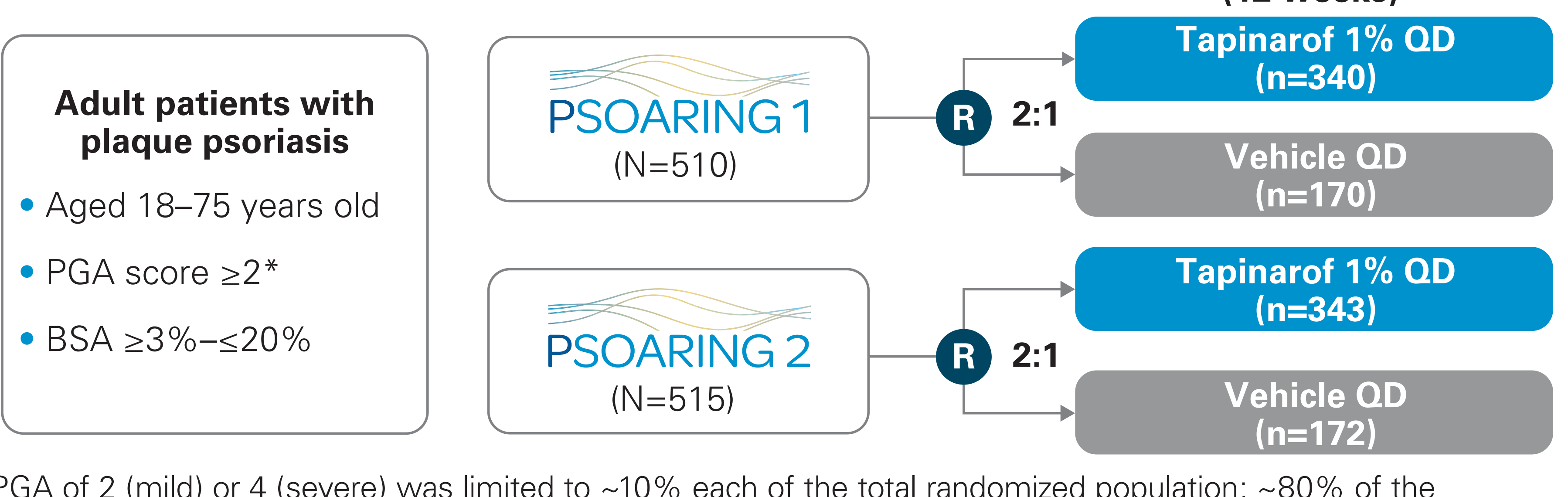

\section{*PGA of 2 (mild) or 4 (severe) was limited to $10 \%$
randomized population had a PGA of 3 (moderate).}

Endpoints and Statistical Analysis

The primary endpoint was PGA response at Week 12, defined as the proportion of patients with a PGA score of clear (0) or almost clear (1) and $\geq 2$-grade improvement in PGA score from baseline to Week 12

The incidence, frequency, and nature of adverse events (AEs) and serious AEs were monitored from the start of study treatment until the end-of-study visit

- The pooled analyses used multiple imputation for the intention-to-treat (ITT) populations in PSOARING 1 and 2

- Tapinarof cream 1\% OD and vehicle groups were compared within each subgroup for the primary endpoint using 95\% confidence intervals for the relative risk calculated using Cochran-Mantel-Haenszel analyses stratified by baseline PGA score

\section{RESULTS}

Patient Disposition and Baseline Characteristics

The pooled analysis population included 1025 patients randomized to tapinarof cream 1\% QD ( $n=683)$ or vehicle $Q D(n=342)$ in PSOARING 1 and 2 (ITT population)

Baseline disease characteristics and demographics in the pooled population were comparable across treatment groups (Table 1) Overall, at baseline, $82 \%$ had a PGA score of 3 (moderate), 57\% had psoriasis for $>10$ years, and $26 \%$ had $\geq 10 \%$ body surface area (BSA) affected
Table 1. Baseline Patient Demographics and Disease Characteristics (Pooled PSOARING 1 and 2)

\begin{tabular}{|c|c|c|}
\hline & $\begin{array}{c}\text { Tapinarof 1\% QD } \\
(n=683)\end{array}$ & $\begin{array}{c}\text { Vehicle QD } \\
\text { (n=342) }\end{array}$ \\
\hline Age $<65$ years, $\mathbf{n}(\%)$ & $584(85.5)$ & $302(88.3)$ \\
\hline Male, n (\%) & $401(58.7)$ & $188(55.0)$ \\
\hline \multicolumn{3}{|l|}{ Race, n (\%) } \\
\hline Caucasian & $586(85.8)$ & $284(83.0)$ \\
\hline Asian & $46(6.7)$ & $25(7.3)$ \\
\hline Black/African American & $30(4.4)$ & $17(5.0)$ \\
\hline American Indian or Alaska Native & $2(0.3)$ & $2(0.6)$ \\
\hline Native Hawaiian or Other Pacific Islander & $1(0.1)$ & $1(0.3)$ \\
\hline Other* & $13(1.9)$ & $10(2.9)$ \\
\hline \multicolumn{3}{|l|}{ Country of enrollment, $\mathbf{n}(\%)$} \\
\hline US & $514(75.3)$ & $262(76.6)$ \\
\hline Canada & $169(24.7)$ & $80(23.4)$ \\
\hline \multicolumn{3}{|l|}{ PGA, n (\%) } \\
\hline 2 - Mild & $67(9.8)$ & 36 (10.5) \\
\hline 3-Moderate & 559 (81.8) & $277(81.0)$ \\
\hline 4-Severe & $57(8.3)$ & $29(8.5)$ \\
\hline \multicolumn{3}{|l|}{ BSA affected, n (\%) } \\
\hline$<10 \%$ & 505 (73.9) & $257(75.1)$ \\
\hline$\geq 10 \%$ & $178(26.1)$ & 85 (24.9) \\
\hline \multicolumn{3}{|l|}{ Duration of disease, $\mathbf{n}(\%)$} \\
\hline$<5$ years & $154(22.5)$ & $73(21.3)$ \\
\hline 5-10 years & $128(18.7)$ & $89(26.0)$ \\
\hline$>10$ years & $401(58.7)$ & $180(52.6)$ \\
\hline
\end{tabular}

PGA Response by Baseline Disease Characteristics and Demographics

The primary endpoint (PGA of 0 or 1 and $\geq 2$-grade improvement at Week 12) was met; PGA response rates were highly statistically significant in the tapinarof cream 1\% OD group versus the vehicle group in both PSOARING 1 and 2: $35.4 \%$ vs $6.0 \%(P<0.0001)$ and $40.2 \%$ vs $6.3 \%(\mathrm{P}<0.0001)$, respectively

The efficacy of tapinarof $1 \%$ QD was consistent across subgroups, regardless of baseline disease characteristics such as PGA score BSA affected, and duration of disease, and demographics such as sex, age, race, country of enrollment (Figure 2)

Notably, based upon the definition of PGA response requiring achievement of $P G A=0$ or 1 with $\geq 2$-grade improvement, mild subjects had to achieve complete disease clearance $(P G A=0)$ and severe subjects had to improve a minimum of 3 points, to be responders

Figure 2. PGA Response at Week 12 by Baseline Disease Characteristics (A) and Demographics (B) (Pooled PSOARING 1 and 2)

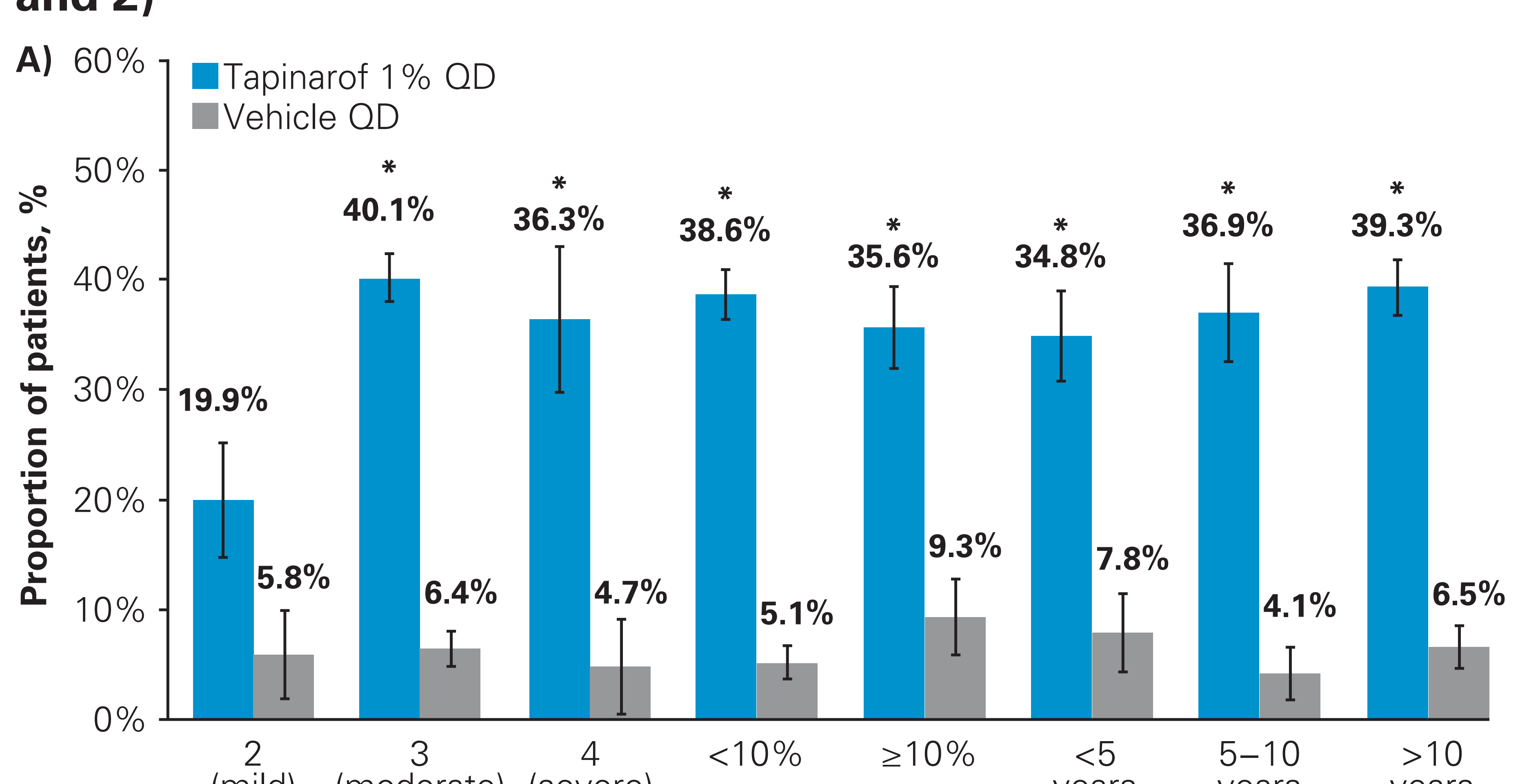
$\begin{array}{ll}5-10 & >10 \\ \text { years } & \text { years }\end{array}$

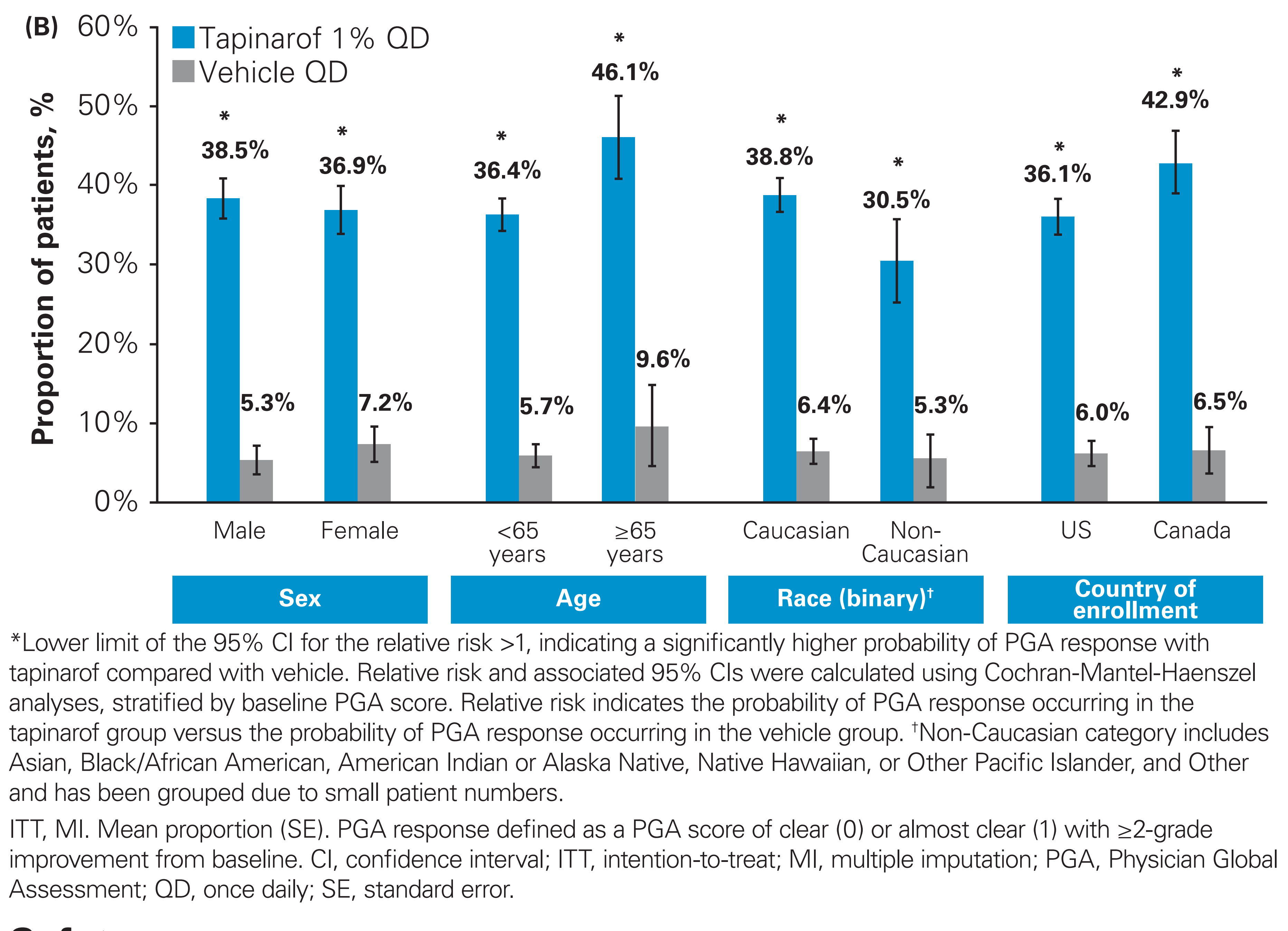

Safety

Treatment-emergent AEs (TEAEs) were mostly mild to moderate in severity

The most common TEAEs overall were folliculitis (20.2\% tapinarof vs $0.9 \%$ vehicle), nasopharyngitis ( $5.7 \%$ tapinarof vs $4.4 \%$ vehicle), and contact dermatitis ( $4.8 \%$ tapinarof vs $0.3 \%$ vehicle)

Although conclusions cannot be drawn due to the small number of patients in some subgroups, the frequency and type of AEs appeared to be generally comparable across subgroups and consistent with those observed in the overall population

\section{CONCLUSIONS}

- Tapinarof cream 1\% QD was consistently efficacious and well tolerated irespective of baseline PGA score, BSA affected, duration of psoriasis, sex, age, race, or country of enrollment (US or Canada)

Due to the small number of patients in some subgroups, limitations exist regarding the ability to draw definitive conclusions from the subgroup analysis

The consistent efficacy and tolerability in all subgroups support the potential use of tapinarof cream 1\% QD across a broad spectrum of disease severity and patient demographics

A long-term extension trial (PSOARING 3) of intermittent treatment with tapinarof $1 \%$ QD based on PGA score demonstrated continued efficacy following the 12-week pivotal trials and an 4-month remittive effect off therapy ${ }^{2}$

- Tapinarof cream 1\% OD has potential to be the first topical psoriasis treatment with a novel mechanism of action in almost 20 years

\section{REFERENCES}

1. Lebwohl M, et al. Skin. 2020;466): 575 , https://doi.org/10.25251/skin.4. supp.75; 2. Strober B, et al. Innovations in
Dermatology Virtual Spring Conference 2021, Poster Presentation, March 16-20, 2021.

\section{ACKNOWLEDGMENTS}

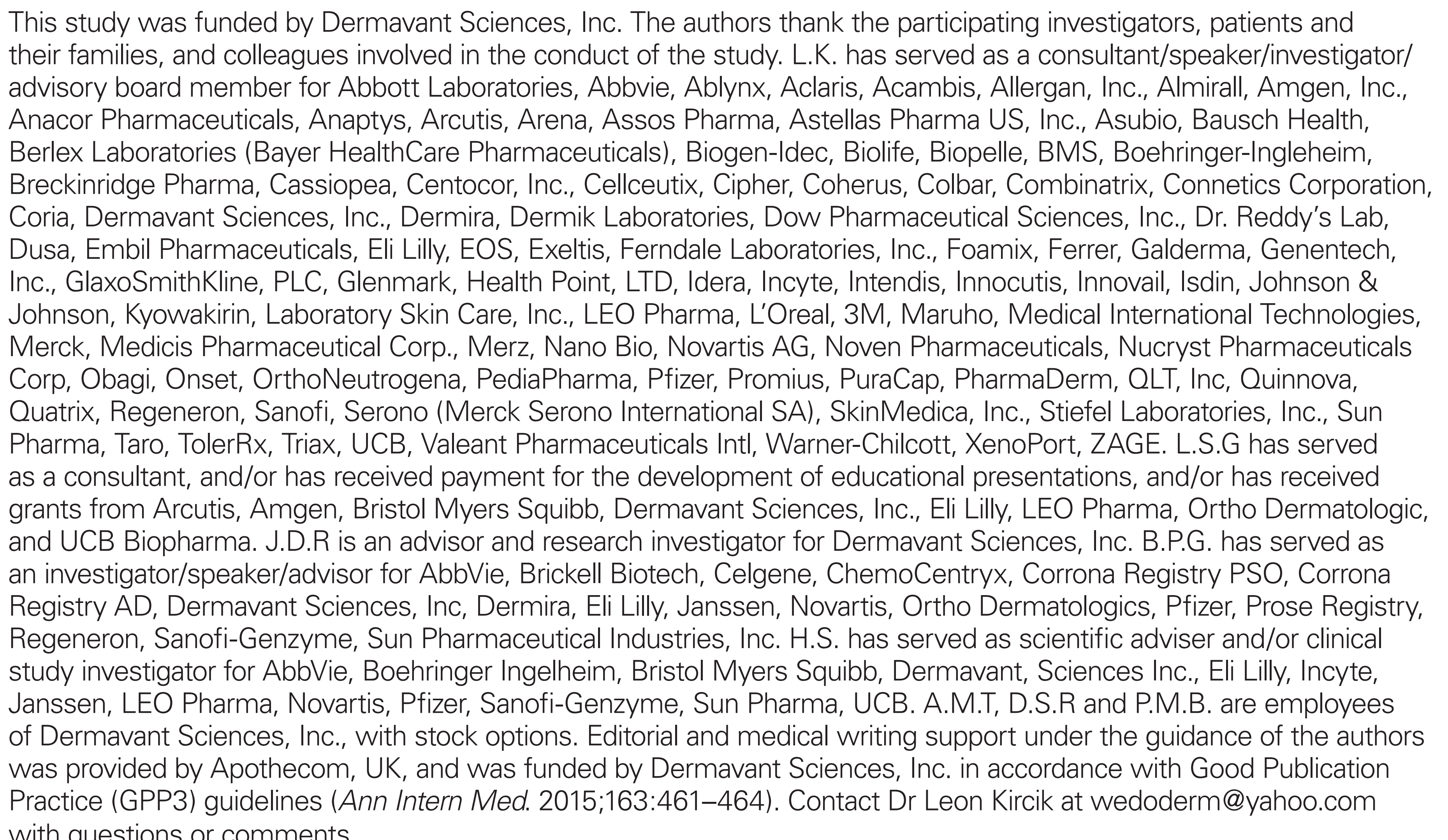

